\title{
Comparison of traditional methods and high-throughput genetic sequencing in the detection of pathogens in pulmonary infectious diseases
}

\author{
Xianqiu Chen ${ }^{\#}$, Kebin Cheng", Xiaoli Sun, Yuan Zhang, Zu Cao, Jianxiong Li, Jiuwu Bai, Haiwen Lu, \\ Shuyi Gu, Li Zhang, Jinfu Xu, Ping Jiang, Shuo Liang \\ Department of Respiratory and Critical Care Medicine, Shanghai Pulmonary Hospital, Tongji University, School of Medicine, Shanghai, China \\ Contributions: (I) Conception and design: S Liang; (II) Administrative support: P Jiang, S Liang; (III) Provision of study materials or patients: K \\ Cheng, X Sun, Y Zhang, Z Cao, J Li, J Bai, H Lu, S Gu, L Zhang, J Xu; (IV) Collection and assembly of data: X Chen, S Liang; (V) Data analysis \\ and interpretation: X Chen, S Liang; (VI) Manuscript writing: All authors; (VII) Final approval of manuscript: All authors. \\ \#These authors contributed equally to this work. \\ Correspondence to: Prof. Shuo Liang, MD; Prof. Ping Jiang, MD. Department of Respiratory and Critical Care Medicine, Shanghai Pulmonary Hospital, \\ Tongji University, School of Medicine, 507 Zheng Min Road, Shanghai 200433, China. Email: Liangshuo79@163.com; jiangpyn67@126.com.
}

\begin{abstract}
Background: The major causes of pulmonary infections are various microorganisms. This study aimed to compare the positive rates of pathogenic microorganism DNA/RNA high-throughput genetic sequencing (PMseq), which is an emerging technique, with traditional methods for pulmonary disease detection, and to investigate the differences in different sample types.

Methods: Bronchoalveolar lavage fluid (BALF) and venous blood samples from 104 patients were collected for detection.

Results: The positive rates of PMseq in BALF and venous blood were both significantly higher than those of traditional methods in the same sample $(\mathrm{P}<0.001)$. For BALF, the detection sensitivities were $96.9 \%$ for non-febrile patients and $100 \%$ for febrile patients. For venous blood, the detection sensitivities were $50.0 \%$ for non-febrile patients and $81.3 \%$ for febrile patients. There was no statistical difference in the sensitivity of venous blood samples with or without fever $(\mathrm{P}=0.075)$. For patients without fever, the sensitivity of BALF was much higher than venous blood samples $(\mathrm{P}<0.001)$. In patients with fever, there were no significant differences between different samples.

Conclusions: This study showed that PMseq has a higher positive rate for the detection of pulmonary diseases. For patients without fever, it is recommended to use BALF instead of venous blood samples because of the higher sensitivity. However, for patients with fever, venous blood samples can be used when bronchoalveolar lavage is inconvenient.
\end{abstract}

Keywords: Pathogenic microorganism DNA/RNA high-throughput genetic sequencing (PMseq); bronchoalveolar lavage fluid (BALF); venous blood; sensitivity; fever

Submitted Mar 03, 2021. Accepted for publication Apr 17, 2021.

doi: $10.21037 / \mathrm{atm}-21-1322$

View this article at: http://dx.doi.org/10.21037/atm-21-1322

\section{Introduction}

The lung is the main organ for gas exchange, transporting oxygen from the atmosphere to the bloodstream and releasing carbon dioxide. During respiration, the lung is continuously exposed to a variety of harmful environmental agents, such as bacteria, viruses, particles, and cigarette

\footnotetext{
$\wedge$ ORCID: 0000-0002-1234-1027.
} 
smoke (1). Chronic or acute exposure to these harmful agents results in damage to the lung $(1,2)$. Pneumonia is an acute infection of the pulmonary parenchyma, accompanied by the presence of acute infiltration on chest radiographs (3). Pulmonary infections have been a cause of high morbidity and mortality, particularly in the geriatric and pediatric populations and in immunocompromised hosts (4).

The major causes of pulmonary infections are microorganisms such as bacteria, fungi, viruses, and parasites. Presently, many diagnostic technologies and tools are used to identify the agents causing infectious diseases (5-7). Speciesspecific polymerase chain reaction (PCR) testing is a widely used method for pathogen detection $(8,9)$. However, this traditional molecular biological detection method requires prior knowledge of pathogen genome sequences, and some unknown pathogens in samples cannot be detected. Thus, it is urgent to develop new sequence-independent detection methods for the identification of these unknown pathogens.

In recent years, the rapid development of next-generation sequencing (NGS) has provided a more comprehensive alternative $(10,11)$. As an emerging technique, NGS has been widely used in medical microbiology due to its highthroughput capabilities, low cost, and rapid turnaround time (12-14). Pathogenic microorganism DNA/RNA highthroughput genetic sequencing (PMseq) could identify 6,868 pathogens based on high-throughput NGS (12). Compared with traditional methods of pathogen detection, PMseq has many advantages, such as the ability to detect non-culturable organisms, relative speed, and almost no prior knowledge of the organisms is required (15). Despite the advantages, there are also some challenges, such as the lack of appropriate reference genomes for comparisons in certain cases and difficulties in sample preparation (15).

In this retrospective study, we intended to observe the sensitivity of PMseq in different samples of patients with pulmonary infections, in order to provide further evidence of its clinical application value.

We present the following article in accordance with the MDAR reporting checklist (available at http://dx.doi. org/10.21037/atm-21-1322).

\section{Methods}

\section{Patients}

A total of 104 patients admitted to the Department of Respiratory and Critical Care Medicine of Shanghai Pulmonary Hospital, Shanghai, China from August 2018 to December 2019 were enrolled. General information including gender, age, and oral temperature at admission were recorded. Patients with an oral temperature over $37.3^{\circ} \mathrm{C}$ were considered febrile patients. The bronchoalveolar lavage fluid (BALF) and venous blood samples were collected for PMseq and traditional culture of pathogens. The study was approved by the Institutional Review Board of Shanghai Pulmonary Hospital (No. K21-227) and written informed consent was obtained from each individual in strict accordance with the principles stipulated in the Declaration of Helsinki (as revised in 2013).

\section{PMseq}

Samples were placed in special containers and stored at a low temperature to be sent to the laboratory as soon as possible. Nucleic acids (including DNA and RNA) were extracted directly from the clinical samples with the QIAamp Viral RNA Mini Kit (QIAGEN). After detection of the concentration and purity of nucleic acids on $1 \%$ agarose gels, the reverse transcription reaction was performed with the PrimeScript RT-PCR Kit to generate single strand cDNA, followed by the synthesis of double strand cDNA using the Second Strand cDNA Synthesis Kit according to the manufacturer's instructions. Then, the double stranded cDNA was disrupted into fragments (200-300 bp). Following end repair, A-tailing, adapter ligation, and the PCR reaction, the cDNA library was constructed using the TruSeq ${ }^{\circledR}$ DNA PCR-Free Sample Preparation Kit (Illumina, San Diego, CA, USA). The library quality was evaluated on the Qubit@ 2.0 Fluorometer (Thermo Scientific, MA, USA) and Agilent Bioanalyzer 2100 system. Finally, the library was sequenced on the Illumina HiSeq 2500 platform after quality control.

The high-quality sequence data was obtained via removing low quality reads, duplication reads, adapter contamination, and removing reads shorter than $35 \mathrm{bp}$. The remaining sequences were mapped to the databases of bacteria, fungi, mycobacterium tuberculosis, parasite, virus, and then we got the results, which were usually obtained within 24 to 48 hours.

Two experienced infectiology experts jointly determined the PMseq results as positive or negative, and whether the patient's PMseq results were consistent with clinical infection. Clinical infection or non-infection was determined comprehensively through the patient's symptoms, signs, chest imaging, and laboratory examinations by another two infectiology experts. This was reevaluated until consensus was reached when disagreements occurred. In addition, a reexamination may be required as appropriate. 
Table 1 Characteristics of enrolled patients

\begin{tabular}{lc}
\hline Characteristics & Statistical description \\
\hline Number of patients & 104 \\
Gender & $73(70.2 \%)$ \\
Male & $31(29.8 \%)$ \\
Female & $53.6 \pm 13.9$ \\
Age (mean \pm SD, year) & \\
Sample type & $74(71.2 \%)$ \\
Bronchoalveolar lavage fluid & $30(28.8 \%)$ \\
Venous blood & \\
Temperature & $75(72.1 \%)$ \\
$\leq 37.3^{\circ} \mathrm{C}$ & $29(27.9 \%)$ \\
$>37.3^{\circ} \mathrm{C}$ & \\
Clinically confirmed infection & $40(38.5 \%)$ \\
No & $64(61.5 \%)$ \\
Yes
\end{tabular}

Data are presented as number of patients with percentage of total in parentheses.

\section{Traditional culture of pathogens}

The enrolled patients simultaneously underwent venous blood or BALF culture for pathogens including bacteria, fungi, and mycobacterium using traditional methods. Among them, only patients with a temperature above $38.5^{\circ} \mathrm{C}$ were examined with venous blood culture, and only patients who had no contraindications for electronic bronchoscopy were examined with BALF culture.

\section{Statistical analysis}

Statistical analysis was performed using SPSS 19.0 software (IBM, Armonk, New York, USA). Continuous variables were presented as mean \pm standard deviation, and classification variables were presented as $\mathrm{n}(\%)$. The chi-square test was used to compare the differences in sensitivity between the 2 groups, and $\mathrm{P}<0.05$ was considered statistically significant.

\section{Results}

\section{Characteristics of the enrolled patients}

Among the 104 patients, there were 73 males (70.2\%) and 31 females $(29.8 \%)$. The clinical data are shown in Table 1 .
The average age of the enrolled patients was $53.6 \pm 13.9$ years. There were 75 patients $(72.1 \%)$ with a temperature above $37.3{ }^{\circ} \mathrm{C}$ and 29 patients $(27.9 \%)$ with a temperature less than $37.3{ }^{\circ} \mathrm{C}$ when admitted to the department. The BALF samples were collected from 74 patients $(71.2 \%)$ and venous blood samples were collected from 30 patients (28.8\%). A total of 64 patients $(61.5 \%)$ had clinically confirmed pulmonary infection while the other patients were not clinically confirmed.

\section{PMseq results for febrile and non-febrile patients}

For non-febrile patients, the PMseq results of BALF samples were positive in 31 infected patients and 30 clinically uninfected patients, and were negative in 1 infected patient and 3 clinically uninfected patients. The PMseq results of venous blood samples were positive in 4 infected patients, and were negative in 4 infected patients and 2 uninfected patients. For febrile patients, 8 were positive and none were negative among the infected patients based on BALF samples. Additionally, 1 was positive and none were negative among the uninfected patients based on BALF samples. For the venous blood samples, 13 were positive and 3 were negative among the infected patients, while 3 were positive and 1 was negative among the uninfected patients (Table 2).

\section{Comparison of positive rates between traditional methods and PMseq}

Positive rates of traditional culture and PMseq in BALF samples are shown in Figure 1A. The positive rates of traditional culture were $5 / 25(20.0 \%)$ and $8 / 56(14.3 \%)$ in patients with and without fever, respectively. The results of venous blood samples are shown in Figure $1 B$. The positive rate of traditional culture was $1 / 28(3.6 \%)$ in patients with fever, while there was no venous blood culture performed in patients without fever. We compared the positive rates between traditional culture and PMseq in these patients. Results showed that the positive rates of PMseq in patients with or without fever were both significantly higher than those of traditional culture in the same sample $(\mathrm{P}<0.001$, respectively).

\section{Detection sensitivity of PMseq}

For BALF samples, the detection sensitivity was $96.9 \%$ (89.1-100\%) for non-febrile patients and $100 \%$ for febrile patients. For venous blood samples, the positive rate was 
Table 2 PMseq results of bronchoalveolar lavage fluid and venous blood in febrile and non-febrile patients

\begin{tabular}{|c|c|c|c|c|}
\hline Sample type & Infection & Non-infection & Sensitivity & $95 \% \mathrm{Cl}$ \\
\hline Positive & 31 & 30 & & \\
\hline Negative & 1 & 3 & & \\
\hline BALF (febrile patients) & & & $100 \%$ & NA \\
\hline Negative & 0 & 0 & & \\
\hline Venous blood (non-febrile patients) & & & $50.0 \%$ & $5.3-94.7 \%$ \\
\hline Positive & 4 & 0 & & \\
\hline Negative & 4 & 2 & & \\
\hline Negative & 3 & 1 & & \\
\hline
\end{tabular}

Data are presented as number of patients. PMseq, pathogenic microorganism DNA/RNA high-throughput genetic sequencing; BALF, bronchoalveolar lavage fluid.
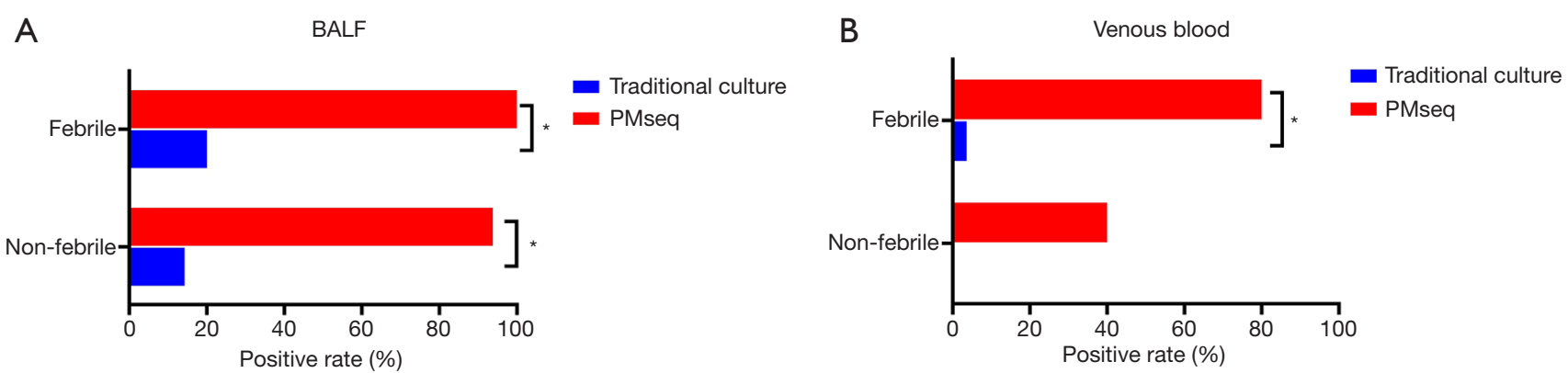

Figure 1 Comparison of positive rates between traditional methods and PMseq. (A) Positive rates of traditional methods and PMseq in BALF samples; (B) positive rates of traditional methods and PMseq in venous blood samples. ${ }^{*} \mathrm{P}<0.001$. Compared with traditional methods, the positive rates of PMseq were significantly increased in both BALF and venous blood samples in patients with or without fever. PMseq, pathogenic microorganism DNA/RNA high-throughput genetic sequencing; BALF, bronchoalveolar lavage fluid.

$50.0 \%(5.3-94.7 \%)$ for non-febrile patients and $81.3 \%(59.8-$ $100 \%)$ for febrile patients. There was no statistical difference between the positive rates of venous blood samples in patients with or without fever $(\mathrm{P}=0.075)$. For patients without fever, the positive rate of BALF was much higher than venous blood samples ( $96.9 \%$ vs. $50 \%, \mathrm{P}<0.001)$. In cases with fever, there was no significant difference between the positive rates of venous blood and BALF samples $(\mathrm{P}=0.280)$ (Figure 2).

\section{Discussion}

The identification of disease-causing pathogens mainly depends on the culture of organisms in the laboratory or the use of specific antibodies and probes based on the sequences of the organism. Nevertheless, these methods are limited in some cases. For example, some microbes are hard to cultivate in the laboratory. For diseases that can be manifested by cultivable organisms, microbiological assays may have a high false negative rate (16). The high false negative rate results in low sensitivity of these assays (17). Metagenomic sequencing is a promising and noninvasive method which involves highthroughput sequencing of complex samples composed of nucleic acids from multiple organisms. When traditional diagnostic assays indicate a novel agent, metagenomic 


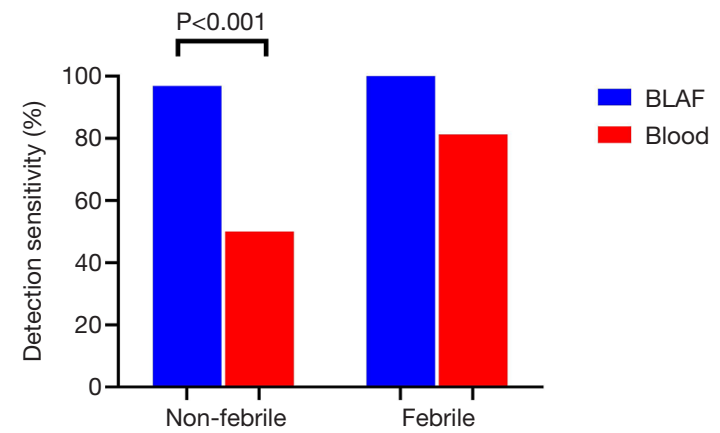

Figure 2 Detection sensitivities of PMseq in different samples. The detection sensitivity of BALF samples was significantly higher than that of venous blood samples in non-febrile patients, $\mathrm{P}<0.001$. PMseq, pathogenic microorganism DNA/RNA high-throughput genetic sequencing; BALF, bronchoalveolar lavage fluid.

sequencing is usually used to follow up (18-20). In this study, we retrospectively analyzed the detection sensitivity of PMseq for detecting pulmonary infections in BALF and venous blood samples of patients with or without fever. Compared with traditional culture, PMseq showed a higher sensitivity in patients with pulmonary diseases. Because PMseq has the advantages of short detection time and wide detection range, it could have certain application value in patients with pulmonary diseases.

Both venous blood and BALF are noninvasive samples used for pulmonary disease detection (21). Different from sputum specimen examination, bronchoalveolar lavage (BAL) has been widely accepted as a powerful tool for pulmonary disease research and has become a standard diagnostic procedure for patients with pulmonary diseases. It is considered to provide additional information for histopathology from biopsy, but has some advantages over biopsy (22). Usually, BAL is performed during fiberoptic bronchoscopy under local anaesthesia (23). However, bronchoscopy is sometimes not convenient for many patients with fever and contraindications. Our study showed that the detection sensitivity of BALF samples was significantly higher than that of venous blood samples for patients without fever. However, for patients with fever, there was no significant difference between the sensitivities of venous blood and BALF samples. Thus, for patients with fever, venous blood samples can be used for detection when BALF samples cannot conveniently be collected.

In conclusion, our study revealed that PMseq has a higher detection sensitivity for pulmonary diseases compared with traditional culture. For patients without fever, BALF is recommended instead of venous blood samples because of the higher sensitivity. However, for patients with fever, venous blood samples can be used, as the detection sensitivity is very close to that of BALF. This study may provide the basis for the further application of PMseq in clinical practice, and contribute to the diagnosis and treatment of pulmonary infectious diseases.

This study has some limitations. It was a retrospective study, and the number of subjects enrolled was not sufficient. Despite this, the results have certain value for clinical work. We will expand the sample size in future work to further verify the conclusions.

\section{Acknowledgments}

Funding: This study was supported by the Fundamental Research Funds for the Central Universities (No. 22120180535) and Dream Mentor-Freshman Training Program of Shanghai Pulmonary Hospital (No. fkxr1901).

\section{Footnote}

Reporting Checklist: The authors have completed the MDARR reporting checklist. Available at http://dx.doi. org/10.21037/atm-21-1322

Data Sharing Statement: Available at http://dx.doi. org/10.21037/atm-21-1322

Conflicts of Interest: All authors have completed the ICMJE uniform disclosure form (available at http://dx.doi. org/10.21037/atm-21-1322). The authors have no conflicts of interest to declare.

Ethical Statement: The authors are accountable for all aspects of the work in ensuring that questions related to the accuracy or integrity of any part of the work are appropriately investigated and resolved. All procedures followed were in accordance with the ethical standards of the responsible committee on human experimentation (institutional and national) and with the Helsinki Declaration (as revised in 2013). The study was approved by the Institutional Review Board of Shanghai Pulmonary Hospital (No. K21-227). Written informed consent was obtained from all patients for being included in the study.

Open Access Statement: This is an Open Access article distributed in accordance with the Creative Commons 
Attribution-NonCommercial-NoDerivs 4.0 International License (CC BY-NC-ND 4.0), which permits the noncommercial replication and distribution of the article with the strict proviso that no changes or edits are made and the original work is properly cited (including links to both the formal publication through the relevant DOI and the license). See: https://creativecommons.org/licenses/by-ncnd/4.0/.

\section{References}

1. Ryter SW, Nakahira K, Haspel JA, et al. Autophagy in pulmonary diseases. Annu Rev Physiol 2012;74:377-401.

2. Bhattacharya J, Matthay MA. Regulation and repair of the alveolar-capillary barrier in acute lung injury. Annu Rev Physiol 2013;75:593-615.

3. Singh D. Imaging of pulmonary infections. Thoracic Imaging: Springer; 2019:147-72.

4. Beigelman-Aubry C, Godet C, Caumes E. Lung infections: the radiologist's perspective. Diagn Interv Imaging 2012;93:431-40.

5. Organization WH. Global status report on noncommunicable diseases 2010. Geneva: WHO; 2011. World Health Organization 2016.

6. Organization WH. Communicable disease alert and response for mass gatherings: key considerations. 2008. Publications of the World Health Organization can be obtained from WHO Press, World Health Organization, Geneva, Switzerland 2008.

7. Kuhn M, Goebel W, Sansonetti PJ. Overview of the bacterial pathogens. Immunology of Infectious Diseases. American Society of Microbiology; 2002:3-23.

8. Ampofo K, Pavia A, Blaschke AJ, et al. Detection of Respiratory Pathogens in Parapneumonic Effusions by Hypothesis-free, Next-Generation Sequencing (NGS). Open Forum Infect Dis 2017;4:S17-S.

9. Hidalgo JA, Alangaden GJ, Eliott D, et al. Fungal endophthalmitis diagnosis by detection of Candida albicans DNA in intraocular fluid by use of a speciesspecific polymerase chain reaction assay. J Infect Dis 2000;181:1198-201.

10. Schuster SC. Next-generation sequencing transforms today's biology. Nat Methods 2008;5:16-8.

11. Van Dijk EL, Auger H, Jaszczyszyn Y, et al. Ten years of next-generation sequencing technology. Trends Genet 2014;30:418-26.

12. Long Y, Zhang Y, Gong Y, et al. Diagnosis of sepsis with cell-free DNA by next-generation sequencing technology in ICU patients. Arch Med Res 2016;47:365-71.

13. Fricke WF, Rasko DA. Bacterial genome sequencing in the clinic: bioinformatic challenges and solutions. Nat Rev Genet 2014;15:49-55.

14. Wilson MR, Naccache SN, Samayoa E, et al. Actionable diagnosis of neuroleptospirosis by next-generation sequencing. N Engl J Med 2014;370:2408-17.

15. Frey KG, Herrera-Galeano JE, Redden CL, et al. Comparison of three next-generation sequencing platforms for metagenomic sequencing and identification of pathogens in blood. BMC Genomics 2014;15:96.

16. Breitkopf C, Hammel D, Scheld HH, et al. Impact of a molecular approach to improve the microbiological diagnosis of infective heart valve endocarditis. Circulation 2005;111:1415-21.

17. Lemmon GH, Gardner SN. Predicting the sensitivity and specificity of published real-time PCR assays. Ann Clin Microbiol Antimicrob 2008;7:18.

18. Towner JS, Sealy TK, Khristova ML, et al. Newly discovered ebola virus associated with hemorrhagic fever outbreak in Uganda. PLoS Pathog 2008;4:e1000212.

19. Palacios G, Lowenstine LJ, Cranfield MR, et al. Human metapneumovirus infection in wild mountain gorillas, Rwanda. Emerg Infect Dis 2011;17:711.

20. Quan PL, Wagner TA, Briese T, et al. Astrovirus encephalitis in boy with $\mathrm{X}$-linked agammaglobulinemia. Emerg Infect Dis 2010;16:918.

21. Skladny H, Buchheidt D, Baust C, et al. Specific detection of Aspergillus species in blood and bronchoalveolar lavage samples of immunocompromised patients by two-step PCR. J Clin Microbiol 1999;37:3865-71.

22. Costabel U, Bonella F, Guzman J. Bronchoalveolar lavage. ERS Handbook of Respiratory Medicine. 2019:138.

23. Picard E, Schwartz S, Goldberg S, et al. A prospective study of fever and bacteremia after flexible fiberoptic bronchoscopy in children. Chest 2000;117:573-7.

(English Language Editor: C. Betlazar-Maseh)

Cite this article as: Chen $\mathrm{X}$, Cheng $\mathrm{K}$, Sun $\mathrm{X}$, Zhang $\mathrm{Y}$, Cao Z, Li J, Bai J, Lu H, Gu S, Zhang L, Xu J, Jiang P, Liang S. Comparison of traditional methods and highthroughput genetic sequencing in the detection of pathogens in pulmonary infectious diseases. Ann Transl Med 2021;9(8):702 doi: 10.21037/atm-21-1322 